QUANTUM PROBABILITY

BANACH CENTER PUBLICATIONS, VOLUME 73

INSTITUTE OF MATHEMATICS

POLISH ACADEMY OF SCIENCES

WARSZAWA 2006

\title{
MULTIPLICATIVE MONOTONE CONVOLUTIONS
}

\author{
UWE FRANZ \\ Département de mathématiques de Besançon \\ Université de Franche-Comté \\ 16, route de Gray, 25030 Besançon Cedex, France \\ E-mail: uwe.franz@math.univ-fcomte.fr
}

\begin{abstract}
Recently, Bercovici has introduced multiplicative convolutions based on Muraki's monotone independence and shown that these convolution of probability measures correspond to the composition of some function of their Cauchy transforms. We provide a new proof of this fact based on the combinatorics of moments. We also give a new characterisation of the probability measures that can be embedded into continuous monotone convolution semigroups of probability measures on the unit circle and briefly discuss a relation to Galton-Watson processes.
\end{abstract}

1. Introduction. In quantum probability there exist several natural notions of independence, see [Mur03] and the references therein. These allow to define new convolutions for probability measure, cf. [VDN92, Voi97, SW97, Mur00].

Bercovici [Ber04] defined multiplicate monotone convolutions for probability measures on the unit circle and on the half line. He showed that with an appropriate function of the Cauchy transform these multiplicative convolutions can be calculated by composition of those functions, similar to Muraki's result [Mur00, Theorem 3.1] for the additive monotone convolution. In this paper we give a new proof of Bercovici's result based on the combinatorics of moments, see Theorem 4.1. Using Berkson and Porta's [BP78] characterization of composition semigroups, one can deduce a characterization of continuous convolution semigroups for the monotone convolution, see [Ber04, Theorem 4.6] or Theorem 6.1 for the case of probability measures on the unit circle.

This paper is organized as follows.

2000 Mathematics Subject Classification: Primary 46L50; Secondary 60E10.

Key words and phrases: monotone independence, conditionally free product, monotone convolutions, Lévy-Khintchine formula.

Work supported in part by the European Community's Human Potential Programme under contract HPRN-CT-2002-00279 QP-Applications and a DAAD-KBN cooperation.

The paper is in final form and no version of it will be published elsewhere. 
In Section 2 we recall the definition of monotone independence and the monotone product of algebraic and quantum probability spaces. In Section 3 we show that the monotone product is actually a special case of the conditionally free product introduced in [BS91, BLS96].

Sections 4, 5, and 6 contain the main results on the multiplicative monotone convolution. We formulate a slightly modified version of a theorem by Bercovici that shows that these convolutions can be calculated by taking the composition of appropriate functions of the Cauchy transform of the measures, see Theorem 4.1 and Corollaries 4.2 and 4.3. We also state a Lévy-Khintchine type characterization of all continuous convolution semigroups for the monotone convolution of probability measures on the unit circle, see Theorem 6.1.

In Section 7, we show that the problem of embedding a probability measure on the unit circle into a continuous monotone convolution semigroup is very similar to the problem of embedding a discrete-time Markovian branching process (or Galton-Watson process) into a continuous-time Markovian branching process. In Section 8 we adapt a characterization of embeddable branching processes due to Goryaĭnov [Gor93] to our situation.

Finally, in the Appendix we discuss the multiplicative monotone convolution of probability measures on the half line and show that there exist two natural, but inequivalent definitions. One of them is equivalent to the definition due to Bercovici and can be treated by similar methods as the multiplicative monotone convolution of measures on the unit circle., cf. [Ber04].

2. Monotone independence. In this section we present the definition of monotone independence and its main properties.

By an algebraic probability space we mean a pair $(\mathcal{A}, \varphi)$ consisting of a unital algebra $\mathcal{A}$ and a unital functional $\varphi: \mathcal{A} \rightarrow \mathbb{C}$. Assume that we have two algebraic probability spaces $\left(\mathcal{A}_{1}, \varphi_{1}\right)$ and $\left(\mathcal{A}_{2}, \varphi_{2}\right)$, such that the first algebra has a decomposition $\mathcal{A}_{1}=\mathbb{C} \mathbf{1} \oplus \mathcal{A}_{1}^{0}$ (direct sum as vector spaces), where $\mathcal{A}_{1}^{0}$ is a subalgebra of $\mathcal{A}_{1}$. Then we define the algebraic monotone product $(\mathcal{A}, \varphi)$ of $\left(\mathcal{A}_{1}, \varphi_{1}\right)$ and $\left(\mathcal{A}_{2}, \varphi_{2}\right)$ as follows, see also [Mur01, Mur03]. The algebra $\mathcal{A}=\mathcal{A}_{1} \amalg \mathcal{A}_{2}$ is the free product of $\mathcal{A}_{1}$ and $\mathcal{A}_{2}$ with identification the units of $\mathcal{A}_{1}$ and $\mathcal{A}_{2}$. The unital functional $\varphi=\varphi_{1} \triangleright \varphi_{2}: \mathcal{A} \rightarrow \mathbb{C}$ is determined by the condition

$$
\varphi\left(b_{1} a_{1} b_{2} \cdots a_{n-1} b_{n}\right)=\varphi_{1}\left(a_{1} \cdots a_{n-1}\right) \varphi_{2}\left(b_{1}\right) \cdots \varphi_{2}\left(b_{n}\right)
$$

for $n \in \mathbb{N}$ and all $a_{1}, \ldots, a_{n-1} \in \mathcal{A}_{1}^{0}, b_{1}, \ldots, b_{n} \in \mathcal{A}_{2}$.

Let now $\mathcal{A}_{1}, \mathcal{A}_{2} \subseteq \mathcal{B}$ be two such algebras, which are contained in an algebraic probability space $(\mathcal{B}, \Phi)$ and denote by $j_{1}: \mathcal{A}_{1} \rightarrow \mathcal{B}, j_{2}: \mathcal{A}_{2} \rightarrow \mathcal{B}$ the inclusion maps. Then the universal property of the free product of algebras implies that there exists a unique homomorphism $j: \mathcal{A}_{1} \amalg \mathcal{A}_{2} \rightarrow B$ such that the following diagram commutes

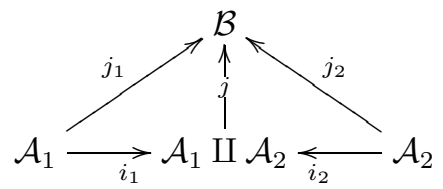

where are $i_{1}: \mathcal{A}_{1} \rightarrow \mathcal{A}_{1} \amalg \mathcal{A}_{2}$ and $i_{2}: \mathcal{A}_{2} \rightarrow \mathcal{A}_{1} \amalg \mathcal{A}_{2}$ are the canonical inclusion maps. 
The subalgebras $\mathcal{A}_{1}, \mathcal{A}_{2}$ are called monotonically independent w.r.t. $\Phi$, if

$$
\Phi \circ j=\left(\Phi \circ j_{1}\right) \triangleright\left(\Phi \circ j_{2}\right)
$$

cf. [Fra02]

We will call a triple $(\mathcal{A}, \mathcal{H}, \Omega)$ consisting of a Hilbert space $\mathcal{H}$, a unit vector $\Omega \in \mathcal{H}$, and a subalgebra $\mathcal{A} \subseteq \mathcal{B}(\mathcal{H})$ a quantum probability space.

If we have an algebraic probability space $(\mathcal{A}, \varphi)$, whose algebra has an involution such that $\Phi$ is even a state, and if for all $a \in \mathcal{A}$ there exists a constant $C_{a} \geq 0$ such that the inequality

$$
\Phi\left(x^{*} a^{*} a x\right) \leq C_{a} \Phi\left(x^{*} x\right)
$$

holds for all $x \in \mathcal{A}$, then the GNS representation $\left(H_{\varphi}, \pi_{\varphi}, \Omega_{\varphi}\right)$ of $(\mathcal{A}, \Phi)$ yields a quantum probability space $\left(\pi_{\varphi}(\mathcal{A}), H_{\varphi}, \Omega_{\varphi}\right)$. If two subalgebras $\mathcal{A}_{1}=\mathbb{C} \mathbf{1} \otimes \mathcal{A}_{1}^{0}, \mathcal{A}_{2} \subseteq \mathcal{A}$ are monotonically independent in $(\mathcal{A}, \varphi)$, then $\pi_{\varphi}\left(\mathcal{A}_{1}^{0}\right)$ and $\pi_{\varphi}\left(\mathcal{A}_{2}\right)$ are monotonically independent in $\left(\pi_{\varphi}(\mathcal{A}), H_{\varphi}, \Omega_{\varphi}\right)$ in the sense of the following definition.

Definition 2.1. Let $\mathcal{H}$ be a Hilbert space, $\Omega \in \mathcal{H}$ a unit vector, and define a state $\Phi: \mathcal{B}(\mathcal{H}) \rightarrow \mathbb{C}$ on the algebra of bounded operators on $\mathcal{H}$ by

$$
\Phi(X)=\langle\Omega, X \Omega\rangle, \quad \text { for } X \in \mathcal{B}(\mathcal{H}) .
$$

Two subalgebras $\mathcal{A}_{1}, \mathcal{A}_{2} \subseteq \mathcal{B}(\mathcal{H})$ are called monotonically independent w.r.t. $\Omega$, if the following two conditions are satisfied.

(a) For all $X, Z \in \mathcal{A}_{1}, Y \in \mathcal{A}_{2}$, we have

$$
X Y Z=\Phi(Y) X Z \text {. }
$$

(b) For all $Y \in \mathcal{A}_{1}, X, Z \in \mathcal{A}_{2}$,

$$
\Phi(X Y Z)=\Phi(X) \Phi(Y) \Phi(Z) .
$$

Two operators $X, Y \in \mathcal{B}(\mathcal{H})$ are called monotonically independent w.r.t. $\Omega$, if the subalgebras $\mathcal{A}_{1}=\operatorname{alg}(X)=\operatorname{span}\left\{X^{k} \mid k=1,2, \ldots\right\}$ and $\mathcal{A}_{2}=\operatorname{alg}(Y)=\operatorname{span}\left\{Y^{k} \mid k=1,2, \ldots\right\}$ are monotonically independent.

Proposition 2.1. Let $\left(\mathcal{A}_{i}, \mathcal{H}_{i}, \Omega_{i}\right), i=1,2$, be two quantum probability spaces, and denote the states associated to $\Omega_{1}$ and $\Omega_{2}$ by $\Phi_{1}$ and $\Phi_{2}$, respectively.

Then there exists a quantum probability space $(\mathcal{A}, \mathcal{H}, \Omega)$ and two injective state-preserving homomorphisms $J_{i}: \mathcal{A}_{i} \rightarrow \mathcal{A}, i=1,2$, such that the images $J_{1}\left(\mathcal{A}_{1}\right)$ and $J_{2}\left(\mathcal{A}_{2}\right)$ are monotonically independent w.r.t. $\Omega$.

Proof. We set $\mathcal{H}=\mathcal{H}_{1} \otimes \mathcal{H}_{2}$ and $\Omega=\Omega_{1} \otimes \Omega_{2}$. Denote by $P_{2}$ the orthogonal projection on $\mathbb{C} \Omega_{2} \subseteq \mathcal{H}_{2}$.

We define the embeddings $J_{i}: \mathcal{A}_{i} \rightarrow \mathcal{B}(\mathcal{H})$ by

$$
\begin{aligned}
& J_{1}(X)=X \otimes P_{2}, \quad \text { for } X \in \mathcal{A}_{1}, \\
& J_{2}(X)=\mathbf{1} \otimes X, \quad \text { for } X \in \mathcal{A}_{2} .
\end{aligned}
$$

For $\mathcal{A}$ we take the subalgebra generated by $J_{1}\left(\mathcal{A}_{1}\right)$ and $J_{2}\left(\mathcal{A}_{2}\right)$. It is clear that $J_{1}$ and $J_{2}$ are injective, state-preserving homomorphism. 
A simple calculation shows that $J_{1}\left(\mathcal{A}_{1}\right)$ and $J_{2}\left(\mathcal{A}_{2}\right)$ are monotonically independent w.r.t. $\Omega$. E.g., for products of the form $J_{1}\left(X_{1}\right) J_{2}(Y) J_{1}\left(X_{2}\right), X_{1}, X_{2} \in \mathcal{A}_{1}, Y \in \mathcal{A}_{2}$, we get

$$
\begin{aligned}
J_{1}\left(X_{1}\right) J_{2}(Y) J_{1}\left(X_{2}\right) & =\left(X_{1} \otimes P_{2}\right)(\mathbf{1} \otimes Y)\left(X_{1} \otimes P_{2}\right)=\left(X_{1} X_{2}\right) \otimes P_{2} Y P_{2} \\
& =\Phi\left(J_{2}(Y)\right) J_{1}\left(X_{1}\right) J_{1}\left(X_{2}\right) .
\end{aligned}
$$

On the other hand, for $J_{2}\left(Y_{1}\right) J_{1}(X) J_{2}\left(Y_{2}\right), X \in \mathcal{A}_{1}, Y_{1}, Y_{2} \in \mathcal{A}_{2}$, we get

$$
\begin{aligned}
\Phi\left(J_{2}\left(Y_{1}\right) J_{1}(X) J_{2}\left(Y_{2}\right)\right) & =\left\langle\Omega_{1} \otimes \Omega_{2},\left(\mathbf{1} \otimes Y_{1}\right)\left(X \otimes P_{2}\right)\left(\mathbf{1} \otimes Y_{2}\right) \Omega_{1} \otimes \Omega_{2}\right\rangle \\
& =\left\langle\Omega_{1} \otimes \Omega_{2}, X \otimes\left(Y_{1} P Y_{2}\right) \Omega_{1} \otimes \Omega_{2}\right\rangle \\
& =\Phi_{1}(X) \Phi_{2}\left(Y_{1}\right) \Phi_{2}\left(Y_{2}\right)=\Phi\left(J_{2}\left(Y_{1}\right)\right) \Phi\left(J_{1}(X)\right) \Phi\left(J_{2}\left(Y_{2}\right)\right) .
\end{aligned}
$$

We will call the quantum probability space $(\mathcal{A}, \mathcal{H}, \Omega)$ constructed in the previous proposition the monotone product of $\left(\mathcal{A}_{1}, \mathcal{H}_{1}, \Omega_{1}\right)$ and $\left(\mathcal{A}_{2}, \mathcal{H}_{2}, \Omega_{2}\right)$. When there is no danger of confusion, we shall identify the algebras $\mathcal{A}_{1}$ and $\mathcal{A}_{2}$ with their images $J_{1}\left(\mathcal{A}_{1}\right)$ and $J_{2}\left(\mathcal{A}_{2}\right)$, respectively.

The monotone product is associative and can be extended to more than two factors, see [Fra01]. But it is not commutative.

The embedding $J_{1}: \mathcal{A}_{1} \rightarrow \mathcal{A}$ is not unital and the product is not trace-preserving. If $\left.\Phi_{1}\right|_{\mathcal{A}_{1}}$ is not identically equal to zero, then the calculation

$$
\Phi_{1}(X) \Phi_{2}\left(Y_{1} Y_{2}\right)=\Phi\left(X Y_{1} Y_{2}\right)=\Phi\left(Y_{2} X Y_{1}\right)=\Phi_{1}(X) \Phi_{2}\left(Y_{1}\right) \Phi_{2}\left(Y_{2}\right)
$$

for all $X \in \mathcal{A}_{1}, Y_{1}, Y_{2} \in \mathcal{A}_{2}$ shows that $\Phi$ can only be a trace on $\mathcal{A}$, if $\left.\Phi_{2}\right|_{\mathcal{A}_{2}}$ is a homomorphism.

3. Relation of monotone independence and conditional free independence. We recall now the definition of the conditional free product of algebraic probability spaces and show that the monotone product is contained as a special case.

Let $\left(\mathcal{A}_{1}, \varphi_{1}, \psi_{1}\right)$ and $\left(\mathcal{A}_{2}, \varphi_{2}, \psi_{2}\right)$ be two unital algebras, equipped with two unital functionals. Recall that the conditionally free product[BS91, BLS96] of $\left(\mathcal{A}_{1}, \varphi_{1}, \psi_{1}\right)$ and $\left(\mathcal{A}_{2}, \varphi_{2}, \psi_{2}\right)$ is defined as the triple $(\mathcal{A}, \varphi, \psi)$, where $\mathcal{A}=\mathcal{A}_{1} \amalg \mathcal{A}_{2}$ is the free product of $\mathcal{A}_{1}$ and $\mathcal{A}_{2}$ with identification the units of $\mathcal{A}_{1}$ and $\mathcal{A}_{2}$. The unital functionals $\varphi$ and $\psi$ on $\mathcal{A}=\mathcal{A}_{1} \amalg \mathcal{A}_{2}$ can be defined by the conditions

$$
\varphi\left(a_{1} a_{2} \cdots a_{n}\right)=\varphi_{\epsilon(1)}\left(a_{1}\right) \cdots \varphi_{\epsilon(n)}\left(a_{n}\right) \quad \text { and } \quad \psi\left(a_{1} a_{2} \cdots a_{n}\right)=0
$$

for all $n \in \mathbb{N}$ and all $a_{i} \in \mathcal{A}_{\epsilon(i)}$ with $\epsilon(i) \in\{1,2\}, \epsilon(1) \neq \epsilon(2) \neq \cdots \neq \epsilon(n)$ and $\psi_{\epsilon(1)}\left(a_{1}\right)=\cdots=\psi_{\epsilon(n)}\left(a_{n}\right)=0$. The functional $\psi$ is simply the free product $\psi_{1} * \psi_{2}$ of $\psi_{1}$ and $\psi_{2}$, cf. [VDN92, Voi97]. We will denote $\varphi$ by

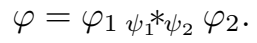

The product defined in this way for triples $(\mathcal{A}, \varphi, \psi)$ can be shown to be commutative and associative, cf. [BS91, BLS96].

Taking pairs of the form $\left(\mathcal{A}_{1}, \varphi_{1}, \varphi_{1}\right)$ and $\left(\mathcal{A}_{2}, \varphi_{2}, \varphi_{2}\right)$, one obtains the free product also for the first functional, i.e.

$$
\varphi_{1 \varphi_{1} \varphi_{2}} \varphi_{2}=\varphi_{1} * \varphi_{2}
$$


Suppose now that the algebras $\mathcal{A}_{1}$ and $\mathcal{A}_{2}$ have decompositions $\mathcal{A}_{i}=\mathbb{C} \mathbf{1} \oplus \mathcal{A}_{i}^{0}, i=1,2$, as a direct sum of vector spaces, such that the $\mathcal{A}_{i}^{0}$ are even subalgebras. If one defines functionals $\delta_{i}: \mathcal{A}_{i} \rightarrow \mathbb{C}$ by

$$
\delta_{i}\left(\lambda \mathbf{1}+a_{0}\right)=\lambda
$$

for $\lambda \in \mathbb{C}, a_{0} \in \mathcal{A}_{i}^{0}, i=1,2$, then one obtains the boolean product

$$
\varphi_{1 \delta_{1} * \delta_{2}} \varphi_{2}=\varphi_{1} \diamond \varphi_{2}
$$

cf. [SW97, BLS96].

Since the conditionally free product of triples of the form $(\mathcal{A}, \varphi, \delta)$ can be shown to be again of the same form, the commutativity and associativity of the boolean product follow immediately from this construction.

One can also obtain the monotone product from the conditionally free product.

Proposition 3.1. Let $\left(\mathcal{A}_{1}, \varphi_{1}\right)$ and $\left(\mathcal{A}_{2}, \varphi_{2}\right)$ be two algebraic quantum probability spaces and assume $\mathcal{A}_{1}$ has a decomposition $\mathcal{A}_{1}=\mathbb{C} \mathbf{1} \oplus \mathcal{A}_{1}^{0}$, where $\mathcal{A}_{1}^{0}$ is a subalgebra of $\mathcal{A}_{1}$. Define a unital functional $\delta_{1}: \mathcal{A}_{1} \rightarrow \mathbb{C}$ as in Equation (3).

Then we have

$$
\varphi_{1} \triangleright \varphi_{2}=\varphi_{1 \delta_{1} \varphi_{2}} \varphi_{2}
$$

Proof. Let $n \in \mathcal{N}, \epsilon(1), \ldots, \epsilon(n) \in\{1,2\}$ such that $\epsilon(1) \neq \epsilon(2) \neq \cdots \neq \epsilon(n)$, and $a_{1} \in \mathcal{A}_{\epsilon(1)}, \cdots, a_{n} \in \mathcal{A}_{\epsilon(n)}$ such that $\delta_{1}\left(a_{k}\right)=0$ if $\epsilon(k)=1$ and $\varphi_{2}\left(a_{k}\right)=0$ if $\epsilon(k)=2$. This implies $a_{k} \in \mathcal{A}_{1}^{0}$ for $\epsilon(k)=1$ and therefore by Equation (1)

$$
\varphi_{1} \triangleright \varphi_{2}\left(a_{1} a_{2} \cdots a_{n}\right)=\varphi_{1}\left(\prod_{k: \epsilon(k)=1} a_{k}\right) \prod_{k: \epsilon(k)=2} \varphi_{2}\left(a_{k}\right)=0
$$

(If the product $a_{1} a_{2} \cdots a_{n}$ does not begin or end with an element of $\mathcal{A}_{2}$, add $\mathbf{1} \in \mathcal{A}_{2}$ in order to apply Equation (1)).

Therefore $\varphi_{1} \triangleright \varphi_{2}$ satisfies condition (2) that defines the conditionally free product $\varphi_{1} \delta_{1}^{*} \varphi_{2} \varphi_{2}$.

With this observation, Muraki's formula [Mur00, Theorem 3.1] for the additive monotone convolution can be deduced from the analytic theory of the additive conditionally free convolution developed in [BLS96].

4. Products of monotonically independent operators. For a bounded operator $X$ in a quantum probability space $(\mathcal{B}(\mathcal{H}), \mathcal{H}, \Omega)$ we define

$$
\psi_{X}(z)=\left\langle\Omega, \frac{z X}{1-z X} \Omega\right\rangle
$$

and

$$
K_{X}(z)=\frac{\psi_{X}(z)}{1+\psi_{X}(z)}
$$

for $|z|<1 /\|X\|$.

The following theorem is similar to [Ber04, Theorem 2.2]. Below we provide a new proof. 
THEOREM 4.1. Let $(B(\mathcal{H}), \mathcal{H}, \Omega)$ be a quantum probability space and $\mathcal{A}_{1}, \mathcal{A}_{2} \subseteq B(\mathcal{H})$ two monotonically independent subalgebras. Let $V_{1}, V_{2} \in \mathbb{C} \mathbf{1}+\mathcal{A}_{1}$, such that $V_{2} V_{1}-\mathbf{1} \in \mathcal{A}_{1}$ and $W \in \mathcal{A}_{2}$. Then we have

$$
K_{V_{1} W V_{2}}(z)=K_{V_{1} V_{2}}\left(K_{W}(z)\right)
$$

for all $|z|<\min \left(1 /\left\|V_{1} W V_{2}\right\|, 1 /\|W\|\right)$.

Proof. Let $M=\max \left(|| V_{1} W V_{2} \|,|| W||\left(|| V_{1} V_{2} \|+2\right)\right)$ and $|z|<1 / M$. Then we have

$$
\begin{aligned}
\frac{z V_{1} W V_{2}}{1-z V_{1} W V_{2}} & =\sum_{n=1}^{\infty}\left(z V_{1} W V_{2}\right)^{n}=\sum_{n=1}^{\infty} z^{n} V_{1} \underbrace{W(X+\mathbf{1}) W \cdots W(X+\mathbf{1})}_{n-1 \text { times }} W V_{2} \\
& =\sum_{n=1}^{\infty} z^{n} \sum_{\substack{k=1 \\
\nu_{1}, \ldots, \nu_{k} \geq 1 \\
\nu_{1}+\cdots+\nu_{k}=n}}^{n} V_{1} W^{\nu_{1}} X W^{\nu_{2}} X \cdots X W^{\nu_{k}} V_{2},
\end{aligned}
$$

where $X=V_{2} V_{1}-\mathbf{1}$.

Using properties (a) and (b) in Definition 2.1, we get

$$
\begin{aligned}
& \psi_{V_{1} W V_{2}}(z)=\left\langle\Omega, \frac{z V_{1} W V_{2}}{1-z V_{1} W V_{2}} \Omega\right\rangle \\
& =\sum_{n=1}^{\infty} z^{n} \sum_{k=1}^{n} \sum_{\substack{\nu_{1}, \ldots, \nu_{k} \geq 1 \\
\nu_{1}+\cdots+\nu_{k}=n}}\left\langle\Omega, V_{1} X^{k-1} V_{2} \Omega\right\rangle\left\langle\Omega, W^{\nu_{1}} \Omega\right\rangle \cdots\left\langle\Omega, W^{\nu_{k}} \Omega\right\rangle \\
& =\sum_{k=1}^{\infty}\left\langle\Omega, V_{1}\left(V_{2} V_{1}-\mathbf{1}\right)^{k-1} V_{2} \Omega\right\rangle\left(\psi_{W}(z)\right)^{k} \\
& =\sum_{k=1}^{\infty}\left\langle\Omega, V_{1} V_{2}\left(V_{2} V_{1}-\mathbf{1}\right)^{k-1} \Omega\right\rangle\left(\psi_{W}(z)\right)^{k} \\
& =\sum_{k=1}^{\infty}\left\langle\Omega, \psi_{W}(z) V_{1} V_{2} \frac{1}{\mathbf{1}-\psi_{W}(z)\left(V_{2} V_{1}-\mathbf{1}\right)} \Omega\right\rangle \\
& =\sum_{k=1}^{\infty}\left\langle\Omega, \frac{\frac{\psi_{W}(z)}{1+\psi_{W}(z)} V_{1} V_{2}}{1-\frac{\psi_{W}(z)}{1+\psi_{W}(z)} V_{1} V_{2}} \Omega\right\rangle=\psi_{V_{1} V_{2}}\left(K_{W}(z)\right) .
\end{aligned}
$$

By uniqueness of analytic continuation, we get

$$
K_{V_{1} W V_{2}}(z)=K_{V_{1} V_{2}}\left(K_{W}(z)\right)
$$

for all $|z|<\min \left(1 /\left\|V_{1} W V_{2}\right\|, 1 /\|W\|\right)$.

COROLlary 4.2. Let $U, V$ be two unitary operators such that $U-\mathbf{1}$ and $V$ are monotonically independent with respect to $\Omega$. Then we have

$$
K_{U V}(z)=K_{V U}(z)=K_{U}\left(K_{V}(z)\right)
$$

for all $|z| \in \mathbb{D}=\{z \in \mathbb{C}:|z|<1\}$.

Corollary 4.3. Let $X, Y$ be two positive operators such that $X-\mathbf{1}$ and $Y$ are monotonically independent with respect to $\Omega$. Then we have

$$
K_{\sqrt{X} Y \sqrt{X}}(z)=K_{X}\left(K_{Y}(z)\right)
$$

for all $|z|<\min (1 /\|\sqrt{X} Y \sqrt{X}\|, 1 /\|Y\|)$. 
5. Multiplicative monotone convolution for probability measures on the unit circle. For a probability measure $\mu$ on $S^{1}$ we define

$$
\psi_{\mu}(z)=\int_{S^{1}} \frac{z x}{1-z x} \mathrm{~d} \mu(x) \quad \text { and } \quad K_{\mu}(z)=\frac{\psi_{\mu}(z)}{1+\psi_{\mu}(z)}
$$

for $z \in \mathbb{D}=\{z \in \mathbb{C}:|z|<1\}$.

We will call $K_{\mu}$ the $K$-transform of $\mu$, it characterizes the measure $\mu$ completely. Furthermore, for a holomorphic function $K: \mathbb{D} \rightarrow \mathbb{D}$ there exists a probability measure $\mu$ on the unit circle $S^{1}$ such that $K=K_{\mu}$ if and only if $K(0)=0$. This follows from the Herglotz representation theorem, the proof is similar to [Fra04, Proposition 3.3].

It is clear that the composition of two K-transforms is again a $\mathrm{K}$-transform of some probability measure on the unit circle. In view of Corollary 4.2 this suggests the following definition.

Definition 5.1. Let $\mu, \nu$ be two probability measures on $S^{1}$, with tranforms $K_{\mu}$ and $K_{\nu}$. Then the unique probability measure $\mu \triangleright \nu$ on $S^{1}$ with

$$
K_{\mu \triangleright \nu}=K_{\mu} \circ K_{\nu}
$$

is called the monotone convolution of $\mu$ and $\nu$.

REMARK 5.1. 1. The monotone convolution is weakly continuous.

2. The monotone convolution is associative, i.e.

$$
(\lambda \triangleright \mu) \triangleright \nu=\lambda \triangleright(\mu \triangleright \nu)
$$

for all $\lambda, \mu, \nu$, but not commutative, i.e., in general $\mu \triangleright \nu \neq \nu \triangleright \mu$.

3. The Dirac measure $\delta_{1}$ at 1 is a two-sided unit, $\delta_{1} \triangleright \mu=\mu \triangleright \delta_{1}=\mu$ for all $\mu$. Right convolution by a Dirac measure $\delta_{x}$ acts as translation, i.e. $\mu \gg \delta_{x}=T_{x} \mu$, where $T_{x}: S^{1} \rightarrow S^{1}$ is defined by $T_{x}(y)=x y$ for $x \in S^{1}$. But $\delta_{x} \triangleright \mu \neq T_{x} \mu$ in general.

4. The monotone convolution is affine in the first argument. Togehter with weak continuity this implies the following formula

$$
\mu \triangleright \nu=\int_{S^{1}} \mathrm{~d} \mu(x) \delta_{x} \triangleright \nu .
$$

6. Lévy-Khintchine formula for monotone convolution semigroups. We call a weakly continuous one-parameter family $\left(\mu_{t}\right)_{t \geq 0}$ of probability measures on the unit circle a continuous monotone convolution semigroup, if

$$
\mu_{0}=\delta_{1} \quad \text { and } \quad \mu_{s} \triangleright \mu_{t}=\mu_{s+t}
$$

for all $s, t \geq 0$. By definition a one-parameter family $\left(\mu_{t}\right)_{t \geq}$ is a continuous monotone convolution semigroup if and only if the $K$-transforms $K_{t}=K_{\mu_{t}}, t \geq 0$ form a continuous semigroup w.r.t. to composition. The continuity of the $K$-transforms is uniform in $z$ on compact sets. Our main tool for characterizing continuous monotone convolution semigroups will be Berkson and Porta's [BP78] characterisation of composition semigroups of holomorphic maps. 
THEOREM 6.1 ([Ber04, Theorem 4.6]). Let $\left(\mu_{t}\right)_{t \geq 0}$ be a weakly continuous family of probability measures on the unit circle, with $K$-transforms $\left(K_{t}\right)_{t \geq 0}$. Then the following are equivalent.

(a) $\left(\mu_{t}\right)_{t \geq 0}$ is a continuous monotone convolution semigroup.

(b) $\left(K_{t}\right)_{t \geq 0}$ is a continuous semigroups w.r.t. to composition.

(c) There exists a holomorphic function $u: \mathbb{D} \rightarrow \mathbb{C}$ with $\Re u(z) \geq 0$ for $z \in \mathbb{D}$ such that $\left(K_{t}\right)_{t \geq 0}$ is the (unique) solution of

$$
\frac{\mathrm{d} K_{t}(z)}{\mathrm{d} t}=-K_{t}(z) u\left(K_{t}(z)\right)
$$

for $z \in \mathbb{D}$ and $t \geq 0$, with initial condition $K_{0}(z)=z$.

Proof. The equivalence between (a) and (b) follows from the definition and the continuity properties of the monotone convolution.

The equivalence between (b) and (c) is an immediate consequence of [BP78, Theorem (3.3)], it suffices to identify the fixed point at zero as the Denjoy-Wolff point of the $K_{t}$.

REMARK 6.2. 1. The function $u$ in (c) can be computed from the derivative of $\left(K_{t}\right)_{t \geq 0}$ in $t=0$ by

$$
u(z)=-\left.\frac{1}{z} \frac{\mathrm{d}}{\mathrm{d} t}\right|_{t=0} K_{t}(z),
$$

we will call it the generator of $\left(K_{t}\right)_{t \geq 0}$.

2. Such a function $u$ has a unique Herglotz representation

$$
u(z)=i b+\int_{S^{1}} \frac{w+z}{w-z} \mathrm{~d} \rho(w),
$$

where $b$ is a real number and $\rho$ a finite measure on $S^{1}$.

7. Relation to Galton-Watson processes. A probability measure $\mu$ on the unit circle is called infinitely divisible w.r.t. to the monotone convolution, if for all $n \in \mathbb{N}$ there exists a probability measure $\mu_{n}$ on the unit circle such that

$$
\mu=\underbrace{\mu_{n} \triangleright \cdots \triangleright \mu_{n}}_{n \text { times }}
$$

Bercovici has shown in [Ber04, Theorem 4.7] that all infinitely divisible probability measures can be embedded into a continuous monotone convolution semigroup, i.e. if $\mu$ is infinitely divisible w.r.t. to the monotone convolution, then there exists a continuous monotone convolution semigroup $\left(\mu_{t}\right)_{t \geq 0}$ such that $\mu=\mu_{t}$ for some $t \geq 0$. And from the previous section it is clear this implies that the $\mathrm{K}$-transform $K_{\mu}$ can be embedded into a continuous composition semigroup of K-transforms.

A similar problem has been studied in the theory of Galton-Watson processes.

Let $X_{n, k}, n, k=1,2, \ldots$ be independent, identically distributed random variables with values in $\mathbb{N}$ with generating function

$$
\varphi(z)=\mathbb{E}\left(z^{X_{n, k}}\right)=\sum_{m=0}^{\infty} p_{m} z^{m} \quad \text { for } z \in \mathbb{D},
$$


where $p_{m}=\mathbb{P}\left(X_{n, k}=m\right)$. Then the associated Galton-Watson process $\left(Y_{n}\right)_{n \geq 0}$ is defined by $Y_{0}=1$, and

$$
Y_{n+1}=\sum_{k=1}^{Y_{n}} X_{n, k}, \quad \text { for } n \geq 1
$$

This process describes the evolution of a population where after each step each individual produces a random number of offspring according to the probabilities $\left(p_{m}\right)_{m \geq 0}$

Its generating functions form a discrete composition semigroup,

$$
\mathbb{E}\left(z^{Y_{n}}\right)=\varphi^{n}(z), \quad \text { for } z \in \mathbb{D}, \quad n \in \mathbb{N} .
$$

If $\mathbb{P}\left(X_{n, k}=0\right)=0$ (i.e. no individual dies without offspring), then $\varphi(0)=0$ and $\varphi$ is the $K$-transform of a probability measure $\mu$ on $S^{1}$. If $\left(Y_{n}\right)_{n \geq 0}$ can be embedded into a continuous-time Markovian branching process (or equivalently, if $\left(\varphi^{n}\right)_{n \geq 0}$ can be embedded into a continuous composition semigroup $\left(\varphi_{t}\right)_{t \geq 0}$ of generating functions), then $\mu$ is infinitely divisible for the monotone convolution and can be embedded into a continuous monotone convolution semigroup. The problem of embedding Galton-Watson processes has been studied by Goryaĭnov [Gor93, Gor00].

ExAmple 7.1. Continuous-time Markovian branching processes with extinction probability 0 can be obtained by choosing infinitesimal offspring probabilities $\lambda_{j} \geq 0$ for $j \geq 2$ such that $\alpha=\sum_{j=2}^{\infty} \lambda_{j}<\infty$, setting

$$
v(z)=\sum_{j=2}^{\infty} \lambda_{j} z^{j}-\alpha z, \quad \text { for }|z| \leq 1,
$$

and solving the differential equation

$$
\frac{\mathrm{d}}{\mathrm{d} t} \varphi_{t}(z)=v\left(\varphi_{t}(z)\right)
$$

with initial condition $\varphi_{0}(z)=z$, cf. [Gor93, Theorem 4].

A simple example is the Yule process, where $v(z)=\alpha\left(z^{k}-z\right)$ and

$$
\varphi_{t}(z)=\frac{z e^{-\alpha t}}{\sqrt[k-1]{1-\left(1-e^{-\alpha(k-1) t}\right) z^{k-1}}}, \quad t \geq 0
$$

for some $k \in \mathbb{N}, k \geq 2$. This process describes a population were the individuals are replaced by $k$ new individuals after an exponentially distributed random time.

\section{On the embedding of probability measures into continuous monotone con-} volution semigroups. [Gor93, Theorem 6] and [Gor93, Theorem 6] characterize probability generating functions that can be embedded into composition semigroups of probability generating functions. In this section we give a similar characterization for Ktransforms of probability measures on the unit circle that can be embedded into continuous monotone convolution semigroups.

Let $\left(K_{t}\right)_{t \geq 0}$ be a continuous composition semigroups of K-transforms. By [BP78], $K_{t}$ is differentiable w.r.t. $t$ and satisfies the differential equation

$$
\frac{\mathrm{d}}{\mathrm{d} t} K_{t}(z)=v\left(K_{t}(z)\right)=v(z) K_{t}^{\prime}(z)
$$


for $t \geq 0, z \in \mathbb{D}$, with $v$ given by

$$
v(z)=\left.\frac{\mathrm{d}}{\mathrm{d} t}\right|_{t=0} K_{t}(z) .
$$

This equation follows from the semigroup property $K_{s+t}=K_{s} \circ K_{t}=K_{t} \circ K_{s}$ by differentiation w.r.t. $s$ at $s=0$.

By Theorem 6.1, the function $v$ is of the form $v(z)=-z u(z)$, with a holomorphic function $u: \mathbb{D} \rightarrow \mathbb{C}$ such that $\Re u(z) \geq 0$ for $z \in \mathbb{D}$.

We will need the following lemma.

Lemma 8.1. Let $u: \mathbb{D} \rightarrow \mathbb{C}, u \not \equiv 0$, be a holomorphic function such that $\Re u(z) \geq 0$ for $z \in \mathbb{D}$ and set $\beta=u(0), v(z)=-z u(z)$ for $z \in \mathbb{D}$.

Then, for all $t \geq 0$, the equation

$$
v(f(z))=v(z) f^{\prime}(z), \quad z \in \mathbb{D},
$$

has a unique solution $f$ with $f^{\prime}(0)=e^{-t \beta}$.

Proof. The proof of this lemma is borrowed from [Gor93, Lemma 2].

Let $\left(K_{t}\right)_{t>0}$ be a composition semigroup of K-transforms with generator $u$. Then all $K_{t}, t \geq 0$ satisfy Equation (5). Furthermore, the differential equation that the $K_{t}$ satisfy, implies

$$
\frac{\mathrm{d}}{\mathrm{d} t} K_{t}^{\prime}(0)=-u(0) K_{z}^{\prime}(0)
$$

and therefore $K_{t}^{\prime}(0)=e^{-t \beta}$, since $K_{0}(z)=z$ and $K_{0}^{\prime}(0)=1$. This proves existence.

Let now $f$ be an arbitrary solution of Equation (5) with $f^{\prime}(0)=e^{-t \beta}$. Since $v$ has no zeros inside $\mathbb{D}$ other than $z=0$, we get $f(0)=0$ by substituting $z=0$ into Equation (5). Differentiation Equation (5) $k$ times, we can calculate the higher derivatives of $f$ at zero from $f^{\prime}(0)=e^{-t \beta}$ and the derivatives of $v$ at zero. This proves uniqueness.

REMARK 8.2. Let $\left(K_{t}\right)_{t \geq 0}$ be the K-transforms of a continuous monotone convolution semigroup $\left(\mu_{t}\right)_{t \geq 0}$ with generator $u$. Then $K_{t}^{\prime}(0)=e^{-t u(0)}$ is the first moment of $\mu_{t}$, i.e.

$$
e^{-t u(0)}=\int_{S^{1}} x \mathrm{~d} \mu_{t}, \quad \text { for } t \geq 0
$$

We come to the main result of this section.

THEOREM 8.3. Let $\mu$ be a probability measure on the unit circle $S^{1}$ that is not concentrated in one point.

Then $\mu$ can be embedded into a continuous monotone convolution semigroup if and only if $K_{\mu}^{\prime}(z) \neq 0$ for all $z \in \mathbb{D}$ and there exists a locally uniform limit

$$
\lim _{n \rightarrow \infty}-\frac{K_{\mu}^{n}(z)}{\left(K_{\mu}^{n}\right)^{\prime}(z)}=v(z),
$$

in $\mathbb{D}$ that is of the form $v(z)=\alpha z u(z)$ with a non-zero constant $\alpha \in \mathbb{C}$ and a holomorphic function $u: \mathbb{D} \rightarrow \mathbb{C}$ such that $\Re u(z) \geq 0$ for $z \in \mathbb{D}$ and $K_{\mu}^{\prime}(0)=e^{-t_{0} u(0)}$ for some $t_{0} \geq 0$.

Proof. The proof of this theorem is similar to that of [Gor93, Theorem 6]. 
Suppose that $\mu$ can be embedded into a continuous monotone convolution semigroup. Then $K_{\mu}$ can be embedded into a composition semigroup of K-transforms $\left(K_{t}\right)_{t \geq 0}$. Therefore all $K_{t}$ are injective and $K_{t}^{\prime}(z) \neq 0$ for all $z \in \mathbb{D}, t \geq 0$, cf. [BP78]. Denote by $u$ the generator of $\left(K_{t}\right)_{t \geq 0}$ and define $v$ by $v(z)=-z u(z)$ for $z \in \mathbb{D}$. By the Denjoy-Wolff theorem we get $\lim _{t \rightarrow \infty} K_{t}(z)=0$ and $\lim _{t \rightarrow \infty} K_{t}^{\prime}(z)=0$ locally uniformly for all $z \in \mathbb{D}$. Therefore

$$
\lim _{t \rightarrow \infty} \frac{v\left(K_{t}(u)\right)}{K_{t}(z)}=v^{\prime}(0)=-u(0) .
$$

With the right-hand-side of Equation (4) this implies

$$
\lim _{n \rightarrow \infty}-\frac{K_{\mu}^{n}(z)}{\left(K_{\mu}^{n}\right)^{\prime}(z)}=\lim _{t \rightarrow \infty}-\frac{K_{t}(z)}{K_{t}^{\prime}(z)}=\lim _{t \rightarrow \infty}-\frac{K_{t}(z) v(z)}{v\left(K_{t}(z)\right)}=-\frac{v(z)}{v^{\prime}(0)}=-z \frac{u(z)}{u(0)} .
$$

The limit is of the form required in the theorem with the constant $\alpha=1 / u(0)$.

To show the converse, let now $K_{\mu}$ be a K-transform satisfying the conditions of the theorem with $v(z)=\alpha z u(z), \alpha$ and $u$ as described in the theorem.

Let $\left(K_{t}\right)_{t \geq 0}$ be the composition semigroup of K-transforms with generator $u$. Then the $K_{t}$ satisfy

$$
v\left(K_{t}(z)\right)=v(z) K_{t}^{\prime}(z), \quad \text { for } t \geq 0, \quad z \in \mathbb{D} .
$$

The conditions of the theorem imply that $K_{\mu}$ is also a solution of the same equation,

$$
v(z)=\lim _{t \rightarrow \infty}-\frac{K_{\mu}^{n+1}(z)}{\left(K_{\mu}^{n+1}\right)^{\prime}(z)}=\lim _{t \rightarrow \infty}-\frac{K_{\mu}^{n}\left(K_{\mu}(z)\right)}{K_{\mu}^{\prime}(z)\left(K_{\mu}^{n}\right)^{\prime}(z)}=\frac{v\left(K_{\mu}(z)\right)}{K_{\mu}^{\prime}(z)} .
$$

The uniqueness in Lemma 8.1 now implies $K_{\mu}=K_{t_{0}}$.

REMARK 8.4. Let $\mu=\delta_{x}$ be concentrated in one point $x=e^{i \varphi} \in S^{1}$. Then we get $\psi_{\delta_{x}}(z)=\frac{x z}{1-x z}$ and $K_{\mu}(z)=e^{i \varphi} z$ and $\mu$ can be embedded into the continuous convolution semigroups $\left(\mu_{t}^{(k)}\right)_{t \geq 0}$ given by $\mu_{t}^{(k)}=\delta_{e^{i t(\varphi+2 \pi k)}}, k \in \mathbb{Z}$.

\section{Appendix: Multiplicative monotone convolution for probability measures} on $\mathbb{R}_{+}$. Just as there are many different ways to define multiplicatively a positive operator from two given positive operators, there are different definitions of multiplicative monotone convolutions of two probability measures $\mu$ and $\nu$ on $\mathbb{R}_{+}$. Two possible choices are to take positive self-adjoint operators $X$ and $Y$, whose distributions are given by $\mu$ and $\nu$, resp., such that $X-\mathbf{1}$ and $Y-\mathbf{1}$ are monotonically independent, and to define the convolution of $\mu$ and $\nu$ as the distributions of $\sqrt{X} Y \sqrt{X}$ or $\sqrt{Y} X \sqrt{Y}$.

By Corollary 4.3 the K-transform of $\sqrt{X} Y \sqrt{X}$ is equal to the composition of the $\mathrm{K}$-transforms of $X$ and $Y$. Therefore this definition is equivalent to the one chosen by Bercovici, cf. [Ber04].

We will show below that choosing the distribution of $\sqrt{Y} X \sqrt{Y}$ as the convolution of the distributions of $X$ and $Y$ leads to an inequivalent definition.

The operators $\sqrt{X} Y \sqrt{X}$ and $\sqrt{Y} X \sqrt{Y}$ have the same spectrum, except for 0 . More precisely, $\sigma(\sqrt{X} Y \sqrt{X}) \backslash\{0\}=\sigma(\sqrt{Y} X \sqrt{Y}) \backslash\{0\}$, since $\sqrt{X} Y \sqrt{X}=A B$ and $\sqrt{Y} X \sqrt{Y}=$ $B A$ with $A=\sqrt{X} \sqrt{Y}$ and $B=\sqrt{Y} \sqrt{X}$.

But the following example shows that, unlike in the free case where one works with tracial states, here the distributions of $\sqrt{X} Y \sqrt{X}$ and $\sqrt{Y} X \sqrt{Y}$ are in general different 
and therefore we have two different multiplicative monotone convolutions for probability measures on $\mathbb{R}_{+}$.

EXAmple 9.1. Consider the positive definite $2 \times 2$-matrix

$$
M(a)=\left(\begin{array}{ll}
1 & a \\
a & 1
\end{array}\right)=\frac{1}{\sqrt{2}}\left(\begin{array}{cc}
1 & 1 \\
1 & -1
\end{array}\right)\left(\begin{array}{cc}
1+a & 0 \\
0 & 1-a
\end{array}\right) \frac{1}{\sqrt{2}}\left(\begin{array}{cc}
1 & 1 \\
1 & -1
\end{array}\right),
$$

with $a \in(0,1)$. Then we have

$$
\left\langle\left(\begin{array}{l}
0 \\
1
\end{array}\right), A^{k}\left(\begin{array}{l}
0 \\
1
\end{array}\right)\right\rangle=\frac{1}{2}\left((1-a)^{k}+(1+a)^{k}\right)
$$

for $k \in \mathbb{N}$, i.e. the distribution of $A$ in the vector state given by $\omega=\left(\begin{array}{l}0 \\ 1\end{array}\right)$ is equal to $\frac{1}{2}\left(\delta_{1-a}+\delta_{1+a}\right)$.

A simple calculation yields

$$
\sqrt{M(a)}=\frac{1}{2}\left(\begin{array}{cc}
\sqrt{1+a}+\sqrt{1-a} & \sqrt{1+a}-\sqrt{1-a} \\
\sqrt{1+a}-\sqrt{1-a} & \sqrt{1+a}+\sqrt{1-a}
\end{array}\right) .
$$

Let $a, b \in(0,1)$ and consider the pair of positive definite matrices

$$
\begin{aligned}
& X=\mathbf{1} \otimes \mathbf{1}+(M(a)-\mathbf{1}) \otimes P_{\omega}=\left(\begin{array}{llll}
1 & 0 & 0 & 0 \\
0 & 1 & 0 & 0 \\
0 & 0 & 1 & a \\
0 & 0 & a & 1
\end{array}\right), \\
& Y=\mathbf{1} \otimes M(b)=\left(\begin{array}{llll}
1 & 0 & b & 0 \\
0 & 1 & 0 & b \\
b & 0 & 1 & 0 \\
0 & b & 0 & 1
\end{array}\right),
\end{aligned}
$$

in $\mathcal{M}_{2}(\mathbb{C}) \otimes \mathcal{M}_{2}(\mathbb{C}) \cong \mathcal{M}_{4}(\mathbb{C})$ where $P_{\omega}$ denotes the orthogonal projection onto $\omega=\left(\begin{array}{l}0 \\ 1\end{array}\right)$. With respect to the vector state given by $\omega \otimes \omega, X-\mathbf{1} \otimes \mathbf{1}$ and $Y-\mathbf{1} \otimes \mathbf{1}$ are monotonically independent, with distributions given by $\frac{1}{2}\left(\delta_{1-a}+\delta_{1+a}\right)$ and $\frac{1}{2}\left(\delta_{1-b}+\delta_{1+b}\right)$, respectively.

As in Equation (6), we compute

$$
\begin{aligned}
\sqrt{X} & =\left(\begin{array}{cccc}
1 & 0 & 0 & 0 \\
0 & 1 & 0 & 0 \\
0 & 0 & \frac{\sqrt{1+a}+\sqrt{1-a}}{2} & \frac{\sqrt{1+a}-\sqrt{1-a}}{2} \\
0 & 0 & \frac{\sqrt{1+a}-\sqrt{1-a}}{2} & \frac{\sqrt{1+a}+\sqrt{1-a}}{2}
\end{array}\right), \\
\sqrt{Y} & =\frac{1}{2}\left(\begin{array}{cccc}
\sqrt{1+b}+\sqrt{1-b} & 0 & \sqrt{1+b}-\sqrt{1-b} & 0 \\
0 & \sqrt{1+b}+\sqrt{1-b} & 0 & \sqrt{1+b}-\sqrt{1-b} \\
\sqrt{1+b}-\sqrt{1-b} & 0 & \sqrt{1+b}+\sqrt{1-b} & 0 \\
0 & \sqrt{1+b}-\sqrt{1-b} & 0 & \sqrt{1+b}+\sqrt{1-b}
\end{array}\right) .
\end{aligned}
$$


The eigenvalues of both $\sqrt{X} Y \sqrt{X}$ and $\sqrt{X} Y \sqrt{X}$ are

$$
\begin{aligned}
& \lambda_{1}=1+\frac{a}{2}+\frac{1}{2} \sqrt{a^{2}+4(1+a) b^{2}}, \\
& \lambda_{2}=1+\frac{a}{2}-\frac{1}{2} \sqrt{a^{2}+4(1+a) b^{2}}, \\
& \lambda_{3}=1-\frac{a}{2}+\frac{1}{2} \sqrt{a^{2}+4(1-a) b^{2}}, \\
& \lambda_{4}=1-\frac{a}{2}-\frac{1}{2} \sqrt{a^{2}+4(1-a) b^{2}},
\end{aligned}
$$

and therefore their distributions have the same support. But their distributions in the vector state $\omega=\left(\begin{array}{l}0 \\ 0 \\ 0 \\ 1\end{array}\right)$ are different. For example their second moments differ,

$$
\begin{aligned}
& \left\langle\omega,(\sqrt{X} Y \sqrt{X})^{2} \omega\right\rangle=1+b^{2}+a^{2}, \\
& \left\langle\omega,(\sqrt{Y} X \sqrt{Y})^{2} \omega\right\rangle=1+b^{2}+\frac{a^{2}}{2}\left(1+\sqrt{1-b^{2}}\right),
\end{aligned}
$$

(recall that we assumed $a \neq 0, b \neq 0$ ).

Acknowledgements. I presented the results of this paper, in particular Theorems 4.1 and 6.1 at the conference "Quantum Probability and Infinite Dimensional Analysis" in Będlewo, Poland, in June 2004. I wish to thank Marek Bożejko and Janusz Wysoczański who indicated reference [Ber04] to me.

I am also indebted to W. Hazod for bringing Goryaĭnov's work [Gor93, Gor00] to my attention.

\section{References}

[Ber04] H. Bercovici, Multiplicative monotone convolution, preprint math.OA/0406488, 2004.

[BLS96] M. Bożejko, M. Leinert, and R. Speicher, Convolution and limit theorems for conditionally free random variables, Pacific J. Math. 175 (1966), 357-388.

[BP78] E. Berkson and H. Porta, Semigroups of analytic functions and composition operators, Michigan Math. J. 25 (1978), 101-115.

[BS91] M. Bożejko and R. Speicher, $\psi$-independent and symmetrized white noises, in: Quantum Probability \& Related Topics, QP-PQ, VI, World Sci., River Edge, NJ, 1991, 219-236.

[Fra01] U. Franz, Monotone independence is associative, Infin. Dimens. Anal. Quantum Probab. Relat. Top. 4 (2001), 401-407.

[Fra02] U. Franz, What is stochastic independence?, in: Quantum Probability and White Noise Analysis, Non-commutativity, Infinite-dimensionality, and Probability at the Crossroads, QP-PQ, XVI, World Sci., Singapore, 2002, 254-274.

[Fra04] U. Franz, Boolean convolution of probability measures on the unit circle, preprint math.FA/0403243, 2004.

[Gor93] V. V. Goryaŭnov, Fractional iteration of probability-generating functions and the embedding of discrete branching processes into continuous ones, Mat. Sb. 184 (1993), 55-74. 
[Gor00] V. V. Goryănov, One-parameter semigroups of analytic functions and a compositional analogue of infinite divisibility, in: Proceedings of the Institute of Applied Mathematics and Mechanics, Vol. 5 (in Russian), Tr. Inst. Prikl. Mat. Mekh. 5, Nats. Akad. Nauk Ukrainy Inst. Prikl. Mat. Mekh., Donetsk, 2000, 44-57.

[Mur00] N. Muraki, Monotonic convolution and monotone Lévy-Hinčin formula, preprint, 2000 .

[Mur01] N. Muraki, Monotone independence, monotone central limit theorem and monotonic law of small numbers, Inf. Dim. Anal. Quant. Probab. Rel. Top. 4 (2001), 39-58.

[Mur03] N. Muraki, The five independences as natural products, Infin. Dimens. Anal. Quantum Probab. Relat. Top. 6 (2003), 337-371.

[SW97] R. Speicher and R. Woroudi, Boolean convolution, in: D. Voiculescu (ed.), Free Probability Theory (Toronto, 1995), Fields Inst. Commun. 12, American Mathematical Society, Providence, RI, 1997, 267-279.

[VDN92] D. Voiculescu, K. Dykema, and A. Nica, Free Random Variables, AMS, 1992.

[Voi97] D. Voiculescu (ed.), Free Probability Theory (Toronto, 1995), Fields Inst. Commun. 12, American Mathematical Society, Providence, RI, 1997. 